\title{
Schwannoma on Cervical Cervices (C2 \& C3): A Case Report
}

\section{Djoko Supriyandono* and Marjono Dwi Wibowo}

\author{
Department of Surgery, Head and Neck Division, \\ Faculty of Medicine Airlangga Univeristy, Surabaya, Indonesia
}

*Corresponding author details: Djoko Supriyandono; dr.djokosupriyandono@gmail.com

\begin{abstract}
Background: Schwannoma is classified as a benign, encapsulated tumor, derived from the perineural cells which are classified as neural cell membranes. About $10 \%$ of Schwannomas has occurred in the head and neck and the incidence of Schwannoma arising from the Cervical C2 nerve roots is extremely rare.

Aims of study: This case report aims to provide a rare case report on Schwannoma located in cervical cervices (C2\& C3).

Case presentation: A 37-year-old female patient with a lump in the left neck for 4 years ago, the first lump appeared as a larger (increasingly large $1 \mathrm{x} 1 \mathrm{~cm}$ ) marbles with no pain in the lump but over the past 4 months the patient felt his legs and arms are weak. Patients have a history of surgical removal of tumors in the back of the neckline by the end of 2016. At first, we estimated the lump that appeared on the left neck of the patient stands alone in the sense that it was not related to the history of the first lump surgery, but when further examination, the tumor was apparently related to the lump that appeared previously who had surgery.

Conclusion: The extracranial Schwannoma in the head and neck areas is a rare neoplasm. The extracranial Schwannoma case is a case that is difficult to diagnose, in addition to good clinical examination, imaging examination, such as CT scan and MRI are an indispensable way of ensuring the diagnosis of an extracranial Schwannoma.
\end{abstract}

Keywords: Schwannoma; upper cervical spine; surgical excision

\section{INTRODUCTION}

Suicide remains a significant public health concern and is Schwannoma or commonly known as Neurilemoma or Neurinoma is a benign tumor of neural tissue sheet formed from Schwan cells that can appear in all parts of the body, including in the head and neck region area that is about 25\% - 45\% of existing Schwannoma cases. Schwannoma is also found in the areas outside of the head and neck, this includes several Cranial Nerves, including N. V, N. VII, N.X, N.XI, N.XII, or Sympathetic Nerves and peripheral nerves.

Approximately $10 \%$ of Schwannomas that occur in the Head and Neck Region mostly come from the Vagus Nervus or Sympathetic Nerves, whereas Schwannoma derived from the $\mathrm{C} 2$ nerve root is very rare $[1,2]$.

Schwannoma's preoperative diagnosis is obtained using Ultra Sonography, Computed Tomography Scan, Magnetic Resonance Imaging, and Fine Needle Aspiration. But diagnosing Schwannoma is not easy, diagnosing A Schwannoma should be based on the patient's clinical state and further examination. The management of the Schwannoma case is to perform surgery. Performing surgery needs to consider several things, including the risks and benefits of the surgery, where preoperative safety and anticipation of post-operative neurologic deficits should be considered carefully [3].

According to Valentino, et al (1998), the best method for removing Schwannoma is by intracapsular enucleation method, where this method has $30 \%$ higher nerve function benefit when compared with overall tumor resection $[2,4]$.
This study aims to provide a rare case report on Schwannoma located in cervical cervices (C2 \& C3).

\section{CASE PRESENTATION}

A 37-year-old woman came to Surgery Clinic at Dr. Soetomo Hospital with a complaint of a lump on the left side of the neck. This lump appeared for 4 years ago with the size of marbles $1 \times 1 \mathrm{~cm}$ and growing slowly and painless. However, in the last four months, the patient felt an enlarged lump the size of a chicken egg $6 \times 5 \times 4 \mathrm{~cm}$ (Figures 1 and 2) and came along with pain and weak leg, tingling sensation from hand up to the back, and sometimes warm sensation. There was no pulsation in the lump, no signs of abscesses on the lumps. Previously patients had undergone surgical removal of a lump in the back of the neck in December 2016 at Dr. Soetomo Hospital with the results of anatomical lump pathology examination is Schwannoma in Cervical 2 and Cervical 3 (C2 - C3). In the operation the patient mounted internal fixation.

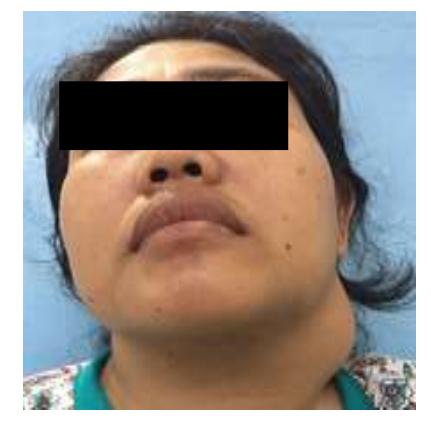

FIGURE 1: preoperative photos 


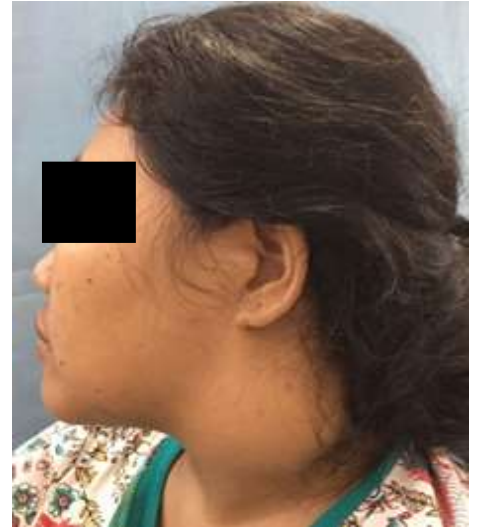

FIGURE 2: preoperative photos

When the patient came to the Surgery clinic at Dr. Soetomo Hospital to check the lump on the left neck of the patient, initially while we assessed with soft tissue tumor. Then, we performed FNAB examination, Coli ultrasound, and Head and Neck CT scan with contrast (Figure 2 and 3). From the FNAB results, it was concluded that a Benign Spindle Mesenchymal Tumor derived from Schwan Cells (Neurofibroma dd Schwannoma), from Coli ultrasound it was concluded as Soft Tissue Mass, whereas MSCT results suggest an image of a neurofibroma with a mass involving the foraminal vertebra as high as VC 2-3 on the left side. This patient was diagnosed with Soft Tissue Mass dd Schwannoma on Regio Coli Sinistra (Figure 1 and 2).

Based on the examination (Figure 3 and 4) we performed, this patient was decided to undergo surgical excision of the tumor with general anesthesia performed together with neurosurgeon due to the position of internal fixation that was performed on the first operation shifted.

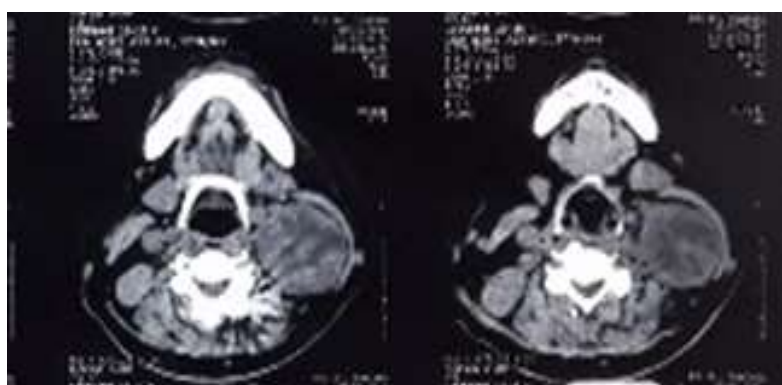

FIGURE 3: CT Scan with contrast

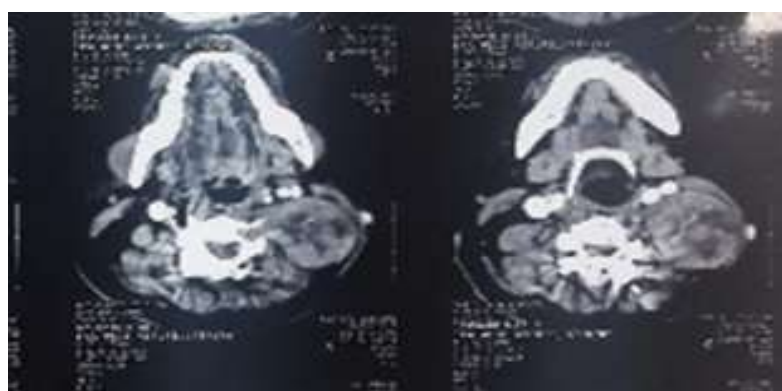

FIGURE 4: CT Scan with contrast

The first stage of surgery was performed by the neurosurgeon in pronation position, followed by head and neck surgeon with supine position, extension of the neck and rotation to the right. Disinfection was using Alcohol $70 \%$. Incision was made to the Submandibular area and deepened layer by layer (Figure 5).

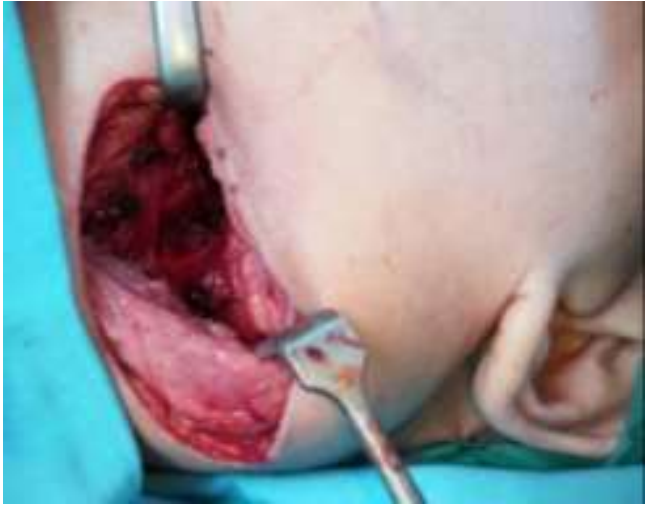

FIGURE 5: Durante operation

Two Tumor Masses were obtained: The first tumor mass in the proximal section was $6 \times 4 \times 2 \mathrm{~cm}$ in size, encapsulated, bluish-white in color, tender, the tip of the first tumor mass associated with a second tumor mass located inferior from the first tumor period (Figure 6).

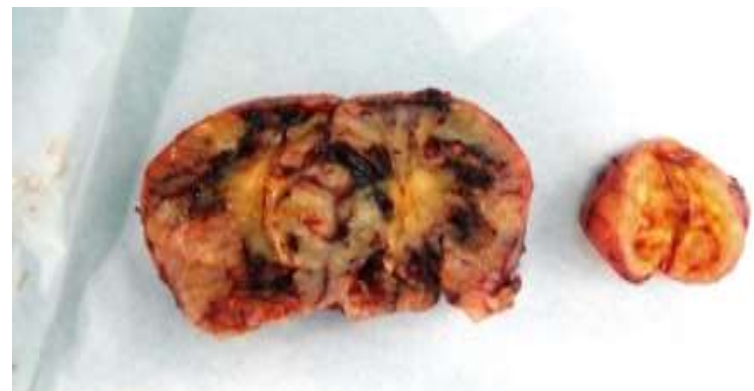

FIGURE 6: Result of tumor excision surgery

The size of the second tumor mass is $3 \times 2 \times 2 \mathrm{~cm}$ tender, yellowish color, the tip of the second Tumor Mass attached to the intervertebral foramen Cervical 2 - 3the management were surgical extirpation of tumors and capsules, bleeding control, primarily repaired layer by layer of surgical wound and placement of redon drain size 12. The results of the operation were examined in Anatomic Pathology Laboratory of Dr. Soetomo Surabaya.

From the laboratory results of Anatomic Pathology, it was found that the lump was well defined tumor, surrounded by fibrous connective tissue, biphasic structure of dense and loose areas. Dense area (Antoni A) consisting of cell proliferation with spindle oval nuclei, fine chromatin, sufficient cytoplasm, no mitosis, some had palisading pattern, forming verocacy bodies. In a loose area (Antoni B) consists of the proliferation of cells with an oval core to spindle, fine chromatin, sufficient cytoplasm, no mitosis. No visible signs of malignancy. From the results of the anatomic pathology reading, it is concluded that the mass present in the patient's left neck is a Schwannoma (Figure 7).

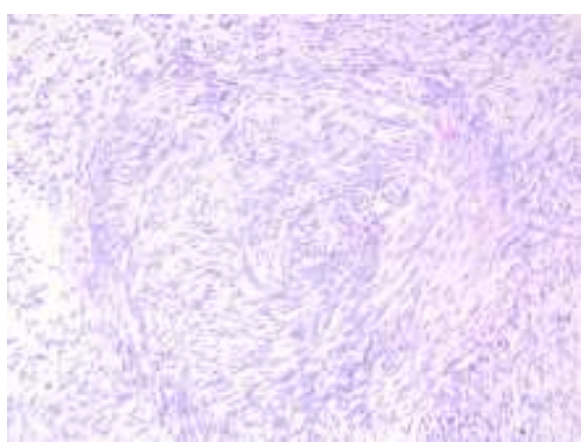

FIGURE 7: Physical pathology of schwannoma cell anatomy (in a circle) 
Our evaluation performed the seventh day postoperatively, surgical wounds healed well without any signs of infection, and no symptoms of neurologic abnormalities were found in the patient (Figure 8).

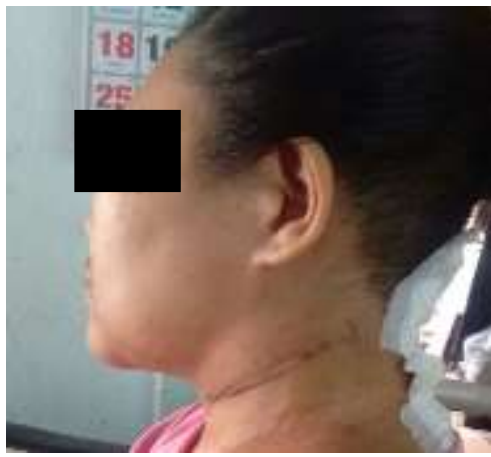

FIGURE 8: Post operation photo after seven days

\section{DISCUSSION}

Schwannoma or commonly known as Neurilemoma or Neurinoma is a benign tumor of neural tissue sheath formed from Schwan cells that can arise in all parts of the body, including in the head and neck region that is about $25 \%-45 \%$ of existing Schwannoma cases. Schwannomas are also found outside the area of head and neck, these include several Cranial Nerves, including N. V, N.XII, or sympathetic nerves and peripheral nerves. Approximately $10 \%$ of Schwannomas that occur in the Head and Neck Region are mostly caused by the Vagus Nervus or Sympathetic Nerves, whereas Schwannoma derived from the $\mathrm{C} 2$ nerve root is very rare $[1,2]$.

Schwannoma Vagal is characterized by difficult to swallow, snoring-like sound, result from Schwannoma derived from sympathetic nerves usually characterized by Horner's Syndrome. In most cases, Schwannoma is difficult to diagnose, the most troubling factor is the difficulty of determining the origin of nerve cells that grow into Schwannoma cells only by physical examination alone [2].

In this case, the tumor that appears on the patient's neck is a continuation of a lump present in C2 and C3 which in 2016 had been done a surgery. Examination especially CT scan is very helpful in establishing the diagnosis and knowing where the origin of the tumor originated, so we will not make mistakes during the surgery [1] Investigations such as MRI are helpful in identifying tumors and knowing the association of tumors with surrounding tissue, either between tumors and other vascular structures, muscles, or nerves around the tumor. Most Schwannomas are not encapsulated. In some cases where nerve fibers surround the tumor, intracapsular enucleation may be performed by preserving the nerves. Based on a study conducted by Valentino, et al (1998), intracapsular enucleation by preserving the nerves or nerve fibers that exist around the tumor can save the nerves function around the tumor by $30 \%$ when compared with cutting the tumor directly [4]. Based on Zbaren et al. (1999), there was no significant difference in the case of recurrence between Schwannoma treatment by direct tumor resection and by intracapsular enucleation [5]. In this case there was no neurological deficit in postoperative patients. Microscopically, Schwannoma is a well-defined tumor, surrounded by fibrous connective tissue, and has biphasic structure of dense and loose areas. Dense area (Antoni A) consisting of cell proliferation with spindle oval nuclei, fine chromatin, sufficient cytoplasm, no mitosis, some had palisading pattern, forming verocacy bodies.

In a loose area (Antoni B) consists of the proliferation of cells with an oval core to spindle, fine chromatin, sufficient cytoplasm, no mitosis. No visible signs of malignancy [6].

\section{CONCLUSION}

The extracranial Schwannoma in the head and neck areas is a rare neoplasm. The extracranial Schwannoma case is a case that is difficult to diagnose, in addition to good clinical examination, imaging examination, such as CT scan and MRI are an indispensable way of ensuring the diagnosis of an extracranial Schwannoma. While examination using FNAB, and histopathologic examination provides a definitive diagnosis. Treatment of this tumor is by surgery, preserving the neural tissues that exist around the tumor. A good surgical technique considering the postoperative neurological deficit impact on Schwannoma tumor is the gold standard for managing a Schwannoma tumor on the head and neck area, so that the functional deficit that can occur can be minimized.

FUNDING: This case report has not been granted any financial assistance from the public, or non-public sources declared

AVAILABILITY OF DATA AND MATERIALS: Not Applicable

DISCLOSURES: Not applicable

AUTHORS'CONTRIBUTIONS: The authors did the conception, design, coordination, drafting, reading, and approval of the final manuscript

ETHICAL APPROVAL: Ethical approval is accepted at Dr. Soetomo General Hospital Ethical committee, Surabaya, Indonesia.

CONSENT FOR PUBLICATION: A written informed consent was obtained from the patient for publication of this case report and any accompanying images.

\section{COMPETING INTERESTS: None declared}

\section{REFERENCES}

[1] Kim SH, Kim NH, Kim KR, Lee JH, Choi H-S (2010) Schwannoma in Head and Neck: Preoperative Imaging Study and Intracapsular Enucleation for Functional Nerve Preservation. Yonsei Med J 51:938. https://doi.org/10.3349/ymj.2010.51.6.938

[2] Singh CV, Shetty S, Deshpande RK, Patel P (2017) Rare cervical nerve root C2-C3 schwannoma. Int $J$ Otorhinolaryngol Head Neck Surg 3:1123. https://doi.org/10.18203/issn.2454-

5929.ijohns20174347

[3] [3] Valentino J, Boggess MA, Ellis JL, Hester TO, Jones RO (1998) Expected neurologic outcomes for surgical treatment of cervical neurilemomas. Laryngoscope 108:1009-1013 . https://doi.org/10.1097/00005537199807000-00011

[4] Zbären P, Markwalder R (1999) Schwannoma of the true vocal cord. Otolaryngol - Head Neck Surg 121:837839. https://doi.org/10.1053/hn.1999.v121.a99282

[5] Das J, saha J (2016) Cervical Schwannoma- A Case Report. Otolaryngology 06:10 $4172 \quad 2161-119$ 1000229

[6] Domanski HA, Walther CS (2017) Nerve Sheath Tumors. In: Miettinen MM (ed) Monographs in Clinical Cytology. Churchill Livingstone, Philadelphia, PA, pp 92-102 